\title{
Research on Power Supply Scheme of Electrical Submersible Pump Fed by Inverter Based on Long Cable
}

\author{
Yonghong Deng ${ }^{1,2}$, Zhishan Liang ${ }^{1}$ \\ ${ }^{1}$ College of Geophysics and Information Engineering, China University of Petroleum Beijing), Beijing, China \\ ${ }^{2}$ School of Electronic and Information engineering, North China Institute of Science and Technology, East Yanjiao, Beijing, China
}

\begin{abstract}
Electrical submersible pump (ESP) require remote operation of inverter via a long cable. The conventional control algorithm of inverter does not operate effectively. In this paper, a new power supply scheme is proposed, namely: Inverter - Motor - Generator - Transformer - Long Cable - ESP power supply program, referred to as IMGEESP power supply system. Based on the analysis of the IMGEESP, the model of the long cable was established, the functional relationship between inverter and smart power of the IMGEESP was deduced, so that the voltage model is constructed. The frequency converter and the intelligent power supply of IMGEESP are coordinated and controlled according to the speed command of the ESP and the cable length. Simulation results verifies the correctness and effectiveness of the proposed IMGEESP.
\end{abstract}

\section{Introduction}

The electric submersible pumps (ESPs) are an important production equipment for oil exploitation in deep-sea oil fields [1]. In the actual production of deep-sea oil fields, this equipment is installed at the up to $7000 \mathrm{~m}$ in depth bottom of subsea oil wells for oil extraction, and is driven by the inverter via long transmission cable of ranging from several kilometers to tens of kilometers [2]. The inverter can control the ESP to a speed that better produces the well and keeps the ESP within its best efficiency range, also offer soft motor starting for ESP systems and reduce impact to power grid during the ESP systems start-up [3]. Hence, the inverter are widely used in oil field facilities, some newer facilities could have up to $80 \%$ loads supplied power by Inverter.

Inverter output is a PWM wave, even if added a sine wave filter, it cannot be completely filtered into a sine wave, but a quasi-sine wave, not to mention when the inverter frequency control, in some frequencies, the filtering effect is very poor, there are it may be the PWM pulse wave output. Inverter output contains harmonic content, the number of harmonics, rich harmonic current content. When the frequency conversion speed control power supply, the inverter output frequency operating point is not fixed, and therefore each frequency has a variety of harmonics, so the harmonics are distributed in a wide frequency domain. Also for the inverter, the power device is used, due to the fast switching action of the power device, its voltage change rate $(\mathrm{du} / \mathrm{dt})$ also generates higher harmonics. Therefore, the inverter and the ESP connection through the long submarine cable incurs some problems $[4,5]$, mainly a travelling wave phenomenon that causes doubling and ringing of the motor terminal voltage, long cable distribution parameters [2] that causes change of the inverter output voltage, current and phase. These problems limit the application of the inverter based on long cable, making the various control schemes of the inverter unable to operate effectively.

In this paper, the new variable frequency power supply scheme, need for long cable, is proposed. The key point of the proposed this scheme is to effectively isolate complex and variable dynamic harmonics and PWM pulse waves, and the output voltage is adjustable and there are no three-phase ACs with harmonics and complete sine wave. The AC power is supplied to the ESP through a long cable.

\section{IMGEESP power supply system composition and principle}

This article proposes a new power supply scheme, as shown in Fig. 1, namely: Inverter - Motor - Generator Transformer - Long Cable - ESP power supply program, referred to as IMGEESP power supply system. This power supply scheme will be more complex variable dynamic harmonics and PWM pulse waves are effectively isolated.

IMGEESP power supply system consists of six parts, including inverter, smart power, synchronous generator set for motor and coupling, detection unit, transformer, etc. When working, the inverter starts according to the requirements of ESP based on long cable, the frequency conversion starter motor, and the synchronous generator set that drives the joint work, and sends out the 
three-phase alternating current of the specified frequency, and generates the constant voltage to frequency ratio alternating current by adjusting the excitation system. The transformer is boosted and supplied to the ESP via a long cable. Through the inverter to achieve speed control, and through the intelligent power supply to achieve voltage control, this proposed power supply system to achieve the frequency conversion speed control of ESP.

The inverter in the IMGEESP power supply system is based on the detection of the three-phase AC frequency, voltage, current and harmonic content emitted by the synchronous generator set. Through its control algorithm and precise torque control, make the IMGEESP output three-phase $\mathrm{AC}$ with stable frequency and high power quality. The intelligent power supply in the IMGEESP power supply system adjusts the three-phase voltage amplitude of its output according to the three-phase AC voltage value of the synchronous generator set detected and the closed-loop excitation control of the synchronous machine, so that the output of the synchronous generator set stabilizes the appropriate three-phase voltage value. The IMGEESP power supply system controls and adjusts the three-phase AC power required by the synchronous generator set to output the ESP according to the variable frequency speed control command, the long cable remote load change requirement and the load current change condition. The IMGEESP power supply system also adjusts the three-phase variable-frequency alternating current output from the synchronous generator set according to the actual operating conditions of the ESP to achieve optimal control.

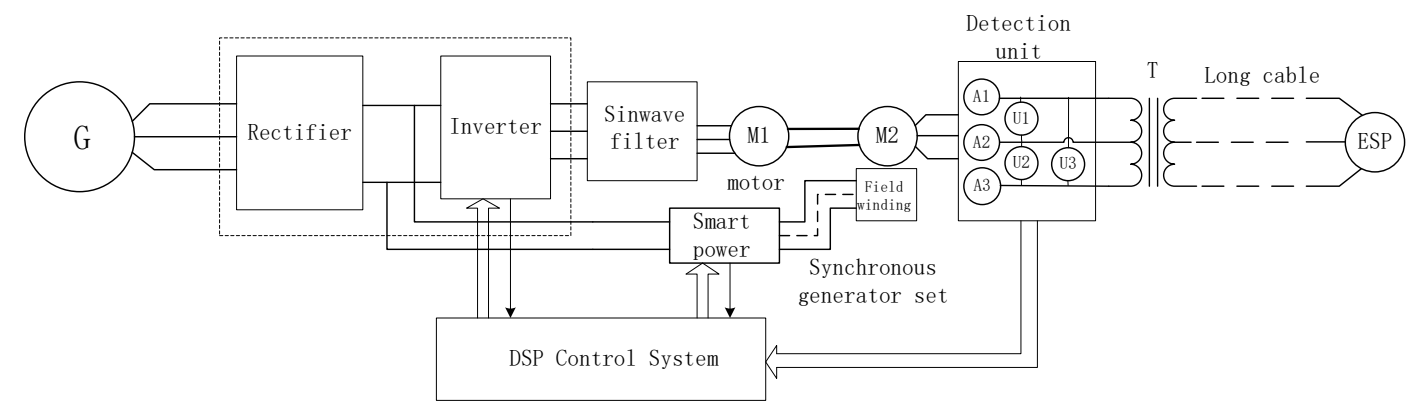

Figure 1. IMGEESP power supply system.

\section{IMGEESP control algorithm implementation}

In the IMGEESP power system, the inverter realizes speed regulation, and the intelligent power supply realizes voltage regulation. The frequency converter and the intelligent power supply are coordinated and controlled according to the speed command of the ESP and the cable length.

\subsection{Long cable modeling and calculation of motor stator voltage}

Thanks to the IMGEESP is sinusoidal output, the voltage and current are as well completely sinusoidal and is solved as a linear equation. Therefore, Lumped $\mathrm{R}$ elements can represent the long cable effectively, even for transient analysis [6], as shown in Fig. 2. As a balanced three-phase network [7], the each phase parameters of long cable model shown in Fig. 2 are equal, and can be transformed to the $\alpha-\beta$ stationary reference frame. There are:

$$
\begin{gathered}
R_{c_{-} u}=R_{c_{-} v}=R_{c_{-} w}=R_{C} \\
u_{c_{-} u v w}=R_{C} * i_{I N V_{-} u v w}
\end{gathered}
$$

where $R_{c_{-} u}, R_{c_{-} v}, R_{C_{-} w}$ are cable resistance per phase $(\Omega) . u_{c_{-} u v w}, i_{I N V_{-} u v w}$ are inverter output voltage drop of the three-phase voltage on the long cable $(\mathrm{V})$, inverter output three-phase current, respectively.

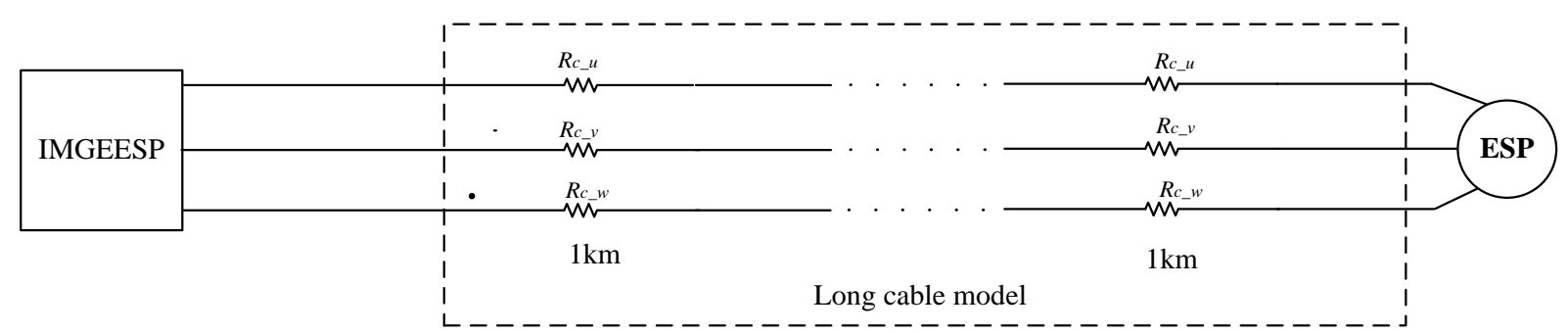

Figure 2. Long cable model of IMGEESP.

\subsection{Algorithm implementation of the IMGEESP}

Fig. 3 shows the vector control algorithm [8] of inverter for the IMGEESP. This algorithm is a speed closed-loop control system. This aims to control and adjustment the frequency of ESP.

Fig. 4 shows control algorithm block diagram of intelligent power supply for the IMGEESP. This 
algorithm is a voltage closed-loop control system. This aims to control and adjustment the voltage of ESP.

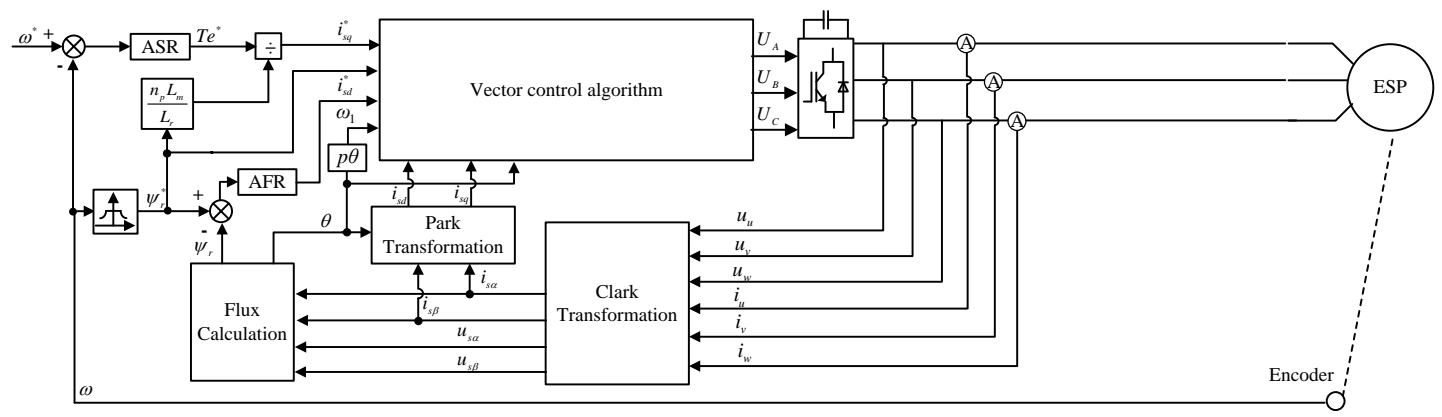

Figure 3. Vector control algorithm of inverter for IMGEESP.

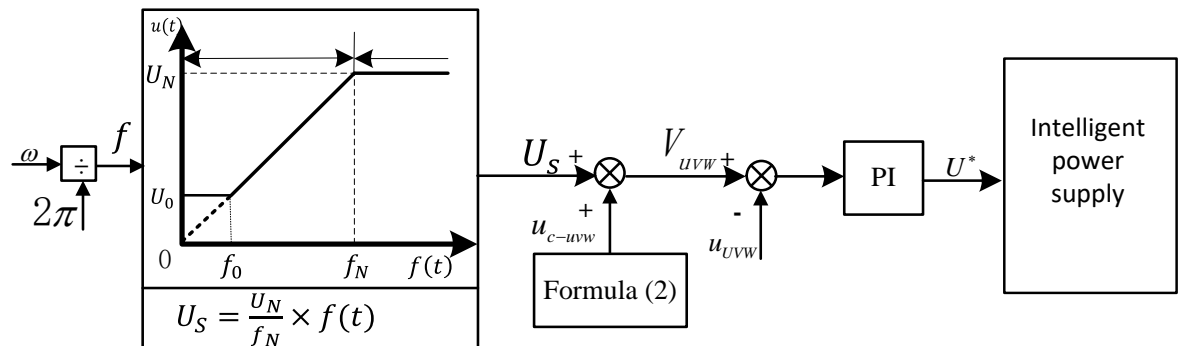

Figure 4. Control algorithm block diagram of intelligent power supply for the IMGEESP.

where $\omega, u_{u v w}$ are angular speed (r/min) and output voltage of synchronous generator set, respectively.

\section{Simulation results}

Computer simulation were performed with the proposed the IMGEESP. The parameters of the long cable and three-phase motor used in simulation are shown in Table 1 and Table 2, and the cable length is $1 \mathrm{~km}$.

Table 1. Long cable parameters.

\begin{tabular}{|c|c|c|}
\hline Quantity & Value & Unit \\
\hline Cable & $\begin{array}{c}\text { 2XS(FL)2YRAA } \\
6 / 10(12) \mathrm{kV}\end{array}$ & - \\
\hline Resistance & 0.34 & $\Omega / \mathrm{km}$ \\
\hline Inductance & 0.38 & $\mathrm{mH} / \mathrm{km}$ \\
\hline Capacitance & 0.29 & $\mu \mathrm{F} / \mathrm{km}$ \\
\hline
\end{tabular}

Table 2. ESP parameters.

\begin{tabular}{|l|l|l|}
\hline Parameter & Value & Unit \\
\hline Rated power & 2.2 & $\mathrm{~kW}$ \\
\hline Rated voltage & $\mathrm{AC} 380$ & $\mathrm{~V}$ \\
\hline Rated current & 5.1 & $\mathrm{~A}$ \\
\hline Rated frequency & 50 & $\mathrm{~Hz}$ \\
\hline Rated speed & 1460 & $\mathrm{rpm}$ \\
\hline
\end{tabular}

Fig. 5 shows simulation waveforms for motor speed and current of the IMGEESP with a $1 \mathrm{~km}$ cable. Fig. 5 shows show that the IMGEESP can control the reliable and stable operation of the motor, provide sufficient starting electromagnetic torque, make the motor start and run smoothly.
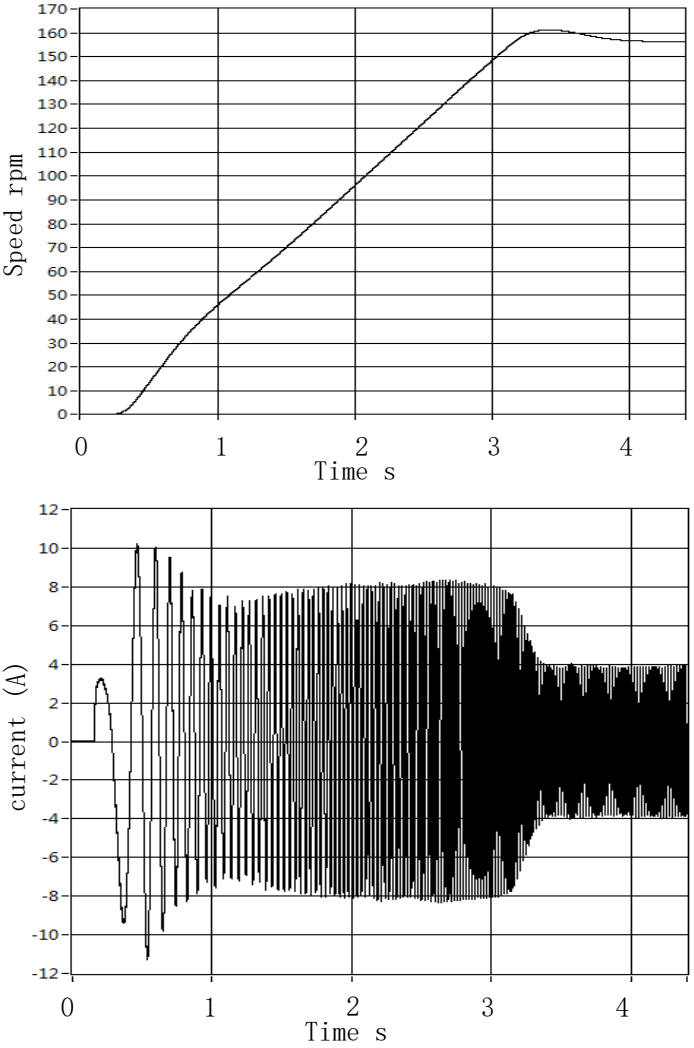

Figure 5. Simulation waveforms for motor speed and current of the IMGEESP with a $1 \mathrm{~km}$ cable.

\section{Conclusion}

In this paper, the proposed IMGEESP that can be effectively applied to long cable-connected motor loads, especially the ESP system. 
1) The IMGESSP output voltage harmonic content is low or no harmonic, no PWM pulse waves interference, there are no complicated working conditions caused by each harmonic and PWM pulse waves interference, making the control more accurate and effective.

2) The IMGEESP increases the service life and efficiency of long cable end loads.

3) The IMGEESP realizes power supply for long-distance variable frequency speed regulation.

\section{Acknowledgments}

School of electronic and information engineering Scientific research infrastructure construction special project (No. 010202580102)

\section{References}

1. C. H. Chien, R. W. G. Bucknall, "Analysis of harmonics in subsea power transmission cables used in VSC-HVDC transmission systems operating under steady-state conditions," IEEE Trans. Power Del., vol. 22, no. 4, pp. 2489-2497, Oct, 2007.

2. Matt Smochek, Anthony F. Pollice, Mukul Rastogi, and Mark Harshman, "Long Cable Applications From a Medium-Voltage Drives Perspective," IEEE TRANSACTIONS ON

INDUSTRY APPLICATIONS, VOL. 52, NO. 1, pp.645-652, JANUARY/FEBRUARY, 2016.
3. Xiaodong Liang, Narayan C. Kar, Joe Liu, "Load Filter Design Method for Medium-Voltage Drive Applications in Electrical Submersible Pump Systems," IEEE TRANSACTIONS ON INDUSTRY APPLICATIONS, VOL.51, NO.3, pp.2017-2029, MAY/JUNE, 2015.

4. H. Abu-Rub, J. Holtz, J. Rodriguez, and G. Baoming, "Medium-voltage multilevel converters-State of the art, challenges, and requirements in industrial applications," IEEE Trans. Ind. Electron., vol. 57, no. 8, pp. 2581-2596, Aug. 2010.

5. A.K. Abdelsalam, M.I. Masoud, S.J. Finney, B.W. Williams, "Vector control PWM-VSI induction motor drive with a long motor feeder: performance analysis of line filter networks", IET Electr. Power, Vol. 5, NO. 5, pp. 443-456, Appl. 2011.

6. S. Amarir and K.Al-Haddad, "A modeling technique to analyze the impact of inverter supply voltage and cable length on industrial motor-drives," IEEE Trans. Power Electron., vol. 23, no. 2, pp. 753-762, Mar. 2008.

7. N. N. Hancock, Matrix Analysis of Electrical Machinery. Amsterdam, The Netherlands: Elsevier, 1975.

8. José Andrés Santisteban, Richard M. Stephan, "Vector Control Methods for Induction Machines: An Overview," IEEE TRANSACTIONS ON EDUCATION, VOL. 44, NO. 2, pp. 170-175, MAY. 2001. 